\title{
Carbon nanotube/polyaniline composite films prepared by in situ electrochemical polymerization for electrochemical supercapacitors
}

\author{
M. Taki ${ }^{\text {a }}$, F. Hekmat ${ }^{\text {a }}$, B. Sohrabi ${ }^{*}$, a M. S. Rahmanifar ${ }^{\text {b }}$ \\ a Department of Chemistry, Surface Chemistry Research Laboratory, Iran University of Science and \\ Technology, P.O. Box 16846-13114, Tehran, Iran. \\ ${ }^{\mathrm{b}}$ Department of Biology, Faculty of basic science, Shahed University, Tehran, Iran. \\ Email: $\underline{\text { Sohrabi_b@iust.ac.ir, sohrabi b@yahoo.com }}$
}

\begin{abstract}
In situ electrochemical polymerization of an aniline solution containing multi-walled carbon nanotube (MWCNT) of 0.8 wt.\% was used to prepare (MWCNT)/polyaniline(PANI) composite films. The electrochemical capacitance performances of these films were investigated with cyclic voltammetry (CV) and ac impedance spectroscopy in $1 \mathrm{M} \mathrm{H}_{2} \mathrm{SO}_{4}$ with a three-electrode system. It was found that the MWCNT/PANI films show much higher specific capacitance (SC), better cyclic stability and more promising for applications in supercapacitors than a pure PANI film electrode. The highest specific capacitance value of 500 $\mathrm{F}^{-1}$ was obtained for the MWCNT/PANI composite film.
\end{abstract}

Keywords: Carbon Nanotube, Polyaniline, Supercapacitors, In Situ Electrochemical Polymerization

\section{Introduction}

Supercapacitors store electrical energy through double layer charging, faradaic processes, or a combination of both. According to the intrinsic principles of charge storage and discharge in supercapacitors, there are two kinds of capacitance: double-layer and pseudocapacitance. The latter involves a faradic process while the former is nonfaradaic[1].

Composites of CNTs and conducting polymer are interesting and promising since they can combine two relatively cheap materials to gain the large pseudocapacitance of the conducting polymers coupled with the conductivity and mechanical strength of the CNTs[1-2]. 
Among various conducting polymers, polyaniline (PANI) is a unique candidate for practical applications due to its good processability, environmental stability, low cost and reversible control of electrical properties by both charge-transfer doping and protonation. However the redox sites in polymer backbone are not sufficiently stable during the many cycled redox processes so, conducting polymers have disadvantages that include lower cycle-life and slow kinetics of ion transport.[3]

Carbon nanotubes have been predicted as excellent filler for polymer on improving the electric conductivity as well as the mechanical properties due to their highly accessible surface area, good electronic properties, low resistivity and high stability.[4]

In fact, composites of conducting polymers and CNTs have been synthesized by either chemical or electrochemical polymerization in the presence of CNTs.

In the chemical approach, an oxidant is needed and converted to a reduction product that may affect the properties of the product. The reaction product is always a powder which means a binder has to be used for the construction of an electrode. The binder is often an insulator and hydrophobic, such as PTFE, and hence inevitably compromises the electrical and electrochemical performance. Electrochemical polymerization has a number of advantages. Particularly, there is no need for added oxidants and electrodeposited conducting polymers are naturally integrated as a continuous uniform film on the electrode, saving the use of a binder.[24]

In this work, We fabricated the MWCNT/PANI composite films for supercapacitor electrode by an in-situ electrochemical polymerization.

\section{Experimental}

\section{1. material preparation}

Multi walled Carbon Nanotubes (MWCNTs) were purchased from Arkema and used as received. In a typical experiment, MWCNTs $(0.8 \mathrm{wt} \%)$ were added to $10 \mathrm{ml}$ of aniline. Then the mixture was heated at reflex for $5 \mathrm{~h}$ in the dark. MWCNT/PANI composite films were deposited on stainless-steel sheet (SS, $0.5 \mathrm{~mm}$ thick) by in-situ electrochemical polymerization. Before deposition, the SS sheet was washed with acetone in ultrasonic bath for 10 minutes and then was dried in air. The area of the electrode used for composite films deposition was $1 \mathrm{~cm}^{2}$. The electrochemical polymerization MWCNT/PANI composite films was carried out by the $\mathrm{CV}$ technique at $100 \mathrm{mVs}^{-1}$ between -0.2 and $-1.2 \mathrm{~V}$ for 100 cycles in a solution of $0.5 \mathrm{M}$ 
$\mathrm{H}_{2} \mathrm{SO}_{4}+0.325 \mathrm{M}$ aniline dissolved MWCNT . The electrochemical polymerization was done in a three-electrode cell with a SS sheet working electrode, a graphite plate counter electrode, and $\mathrm{Ag} / \mathrm{AgCl}$ as a reference electrode. The electro polymerization of aniline dissolved MWCNT can be observed by the color change on the surface of the working electrode. After deposition, the electrode was washed in distilled water and then dried in an oven at $60^{\circ} \mathrm{C}$ for 1 day.

\section{2. Material characterization}

Electrochemical characterizations were carried out by an electrochemical workstation (electroanalyzer system SAMA 500, Iran) in $0.5 \mathrm{M} \mathrm{H}_{2} \mathrm{SO}_{4}$ aqueous solution at room temperature in a three electrode configuration. The electrochemical impedance spectroscopy (EIS) measurements were conducted by a Auto lab PGSTATE 30 model controlled by computer and Nova 1.7 software, applying an alternating current in the frequency range from $10 \mathrm{kHz}$ to $0.1 \mathrm{~Hz}$ with $14 \mathrm{mV}$ amplitude on the Dc voltage of $-0.4 \mathrm{~V}$.

\section{3 .Electropolymerization of MWCNT/PANI composite films:}

The MWCNT/PANI composite films were formed by electro polymerization of aniline dissolved MWCNT. Fig. 1 shows the typical CV growth of MWCNT/PANI film in aqueous $0.5 \mathrm{M} \mathrm{H}_{2} \mathrm{SO}_{4}+0.325 \mathrm{M}$ aniline dissolved with MWCNT of 0.8 wt. $\%$.

\section{Results and discussions}

Continuous potential scanning at $100 \mathrm{mVs}^{-1}$ in the potential range from -0.2 to $-1.2 \mathrm{~V}$ that shows in figure.1 gives a thin uniform green coating on the SS sheet electrode. Peaks correspond to formation of radical cations $(0.3 \mathrm{~V})$, oxidation of head-to-tail dimmer $(0.5 \mathrm{~V})$, and conversion from emeraldine to pernigraniline structure $(0.9 \mathrm{~V})$, respectively [2]. 


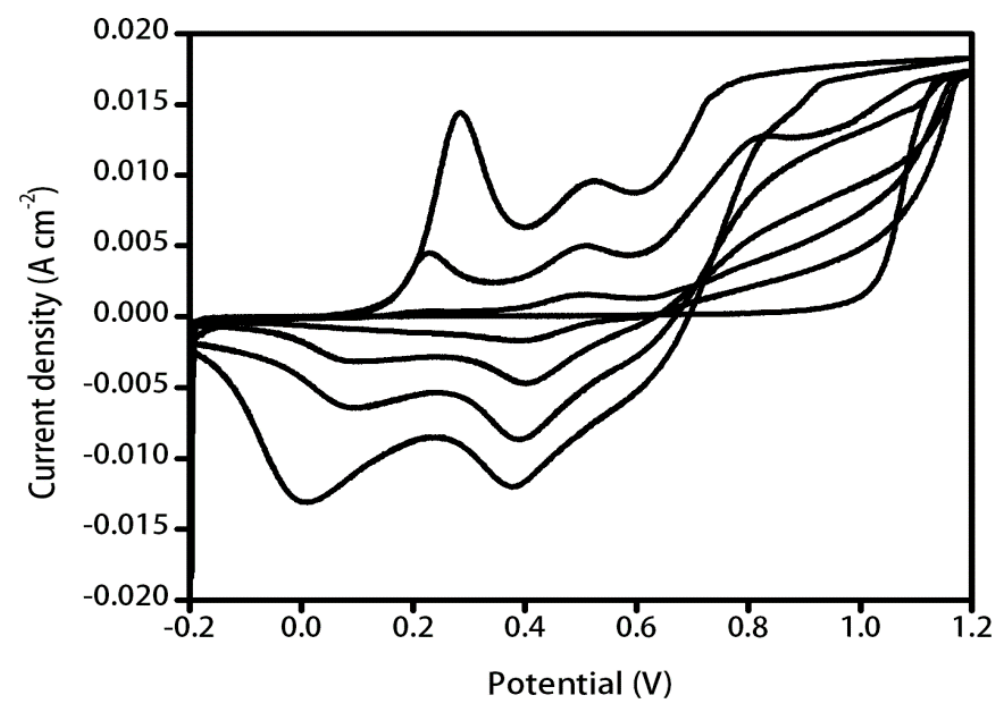

Fig. 1.CVs of growth of PANI-based composite films polymerized from $0.5 \mathrm{M} \mathrm{H}_{2} \mathrm{SO}_{4}$ and $0.325 \mathrm{M}$ aniline solutions with the MWCNT content: $0.8 \mathrm{wt} \%$.

\section{3 .1. Electrochemical response of the MWCNT/PANI composite films}

To elucidate the effect of MWCNT on the properties of PANI films, electrochemical performance of composite films was evaluated by carrying out $\mathrm{CV}$ measurement at $10 \mathrm{mVs}^{-1}$ in $0.5 \mathrm{M} \mathrm{H}_{2} \mathrm{SO}_{4}$ solution. The difference in electrochemical behavior between the pure PANI and MWCNT/PANI composite films is clearly evident from figure 2. The MWCNT/PANI composite films demonstrate strong defined peaks, which indicate high erelectroactivity over pure PANI.

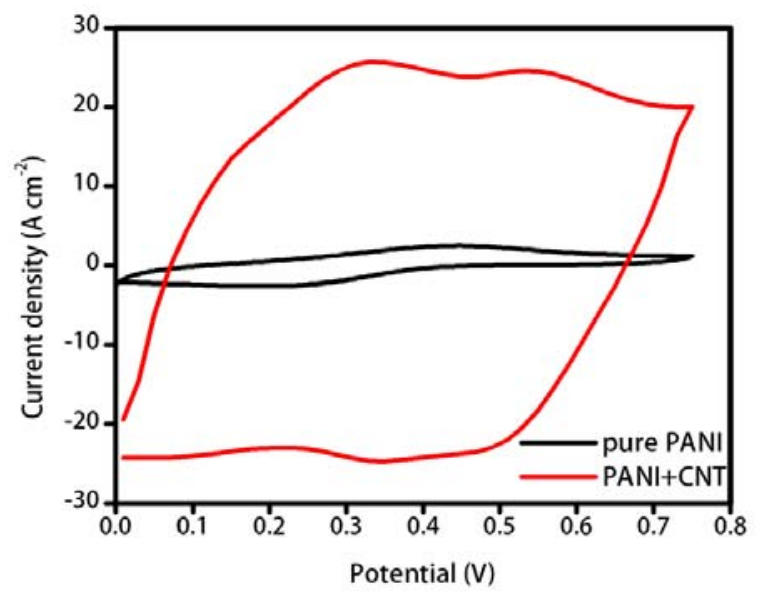

Fig. 2.Voltammetric behavior of the pure PANI and MWCNT/PANI composite films in $0.5 \mathrm{M}$ $\mathrm{H}_{2} \mathrm{SO}_{4}$ electrolyte at $10 \mathrm{mVs}^{-1}$ 
From the Figure 3 , it could be observed that All voltammetric curves on the negative sweeps were very symmetrical to their positive counter parts and this means the excellent reversibility of all these PANI-based films in the high positive potential range, also the CV curves of prepared electrode was kept with increasing scan rate from $20 \mathrm{mV} / \mathrm{s}$ to $100 \mathrm{mV} \mathrm{s}^{-1}$, which could be attributed to the porous structure of the CNT/PANI electrode, which enhanced the accessibility for electrolyte ion transport. As shown in Fig. 3b, the capacitance of prepared electrode decreased slightly upon increasing the scan rate, from $375 \mathrm{~F} \mathrm{~g}^{-1}$ in $20 \mathrm{mV} \mathrm{s}^{-1}$ to 180 $\mathrm{F} \mathrm{g}^{-1}$ in $100 \mathrm{mV} \mathrm{s}^{-1}$, which attributed to diffusion limitations of the electrolyte in the pores of the electrode material. (in lower scan rates specific capacitance arrive to $500 \mathrm{Fg}^{-1}$ ).[5]
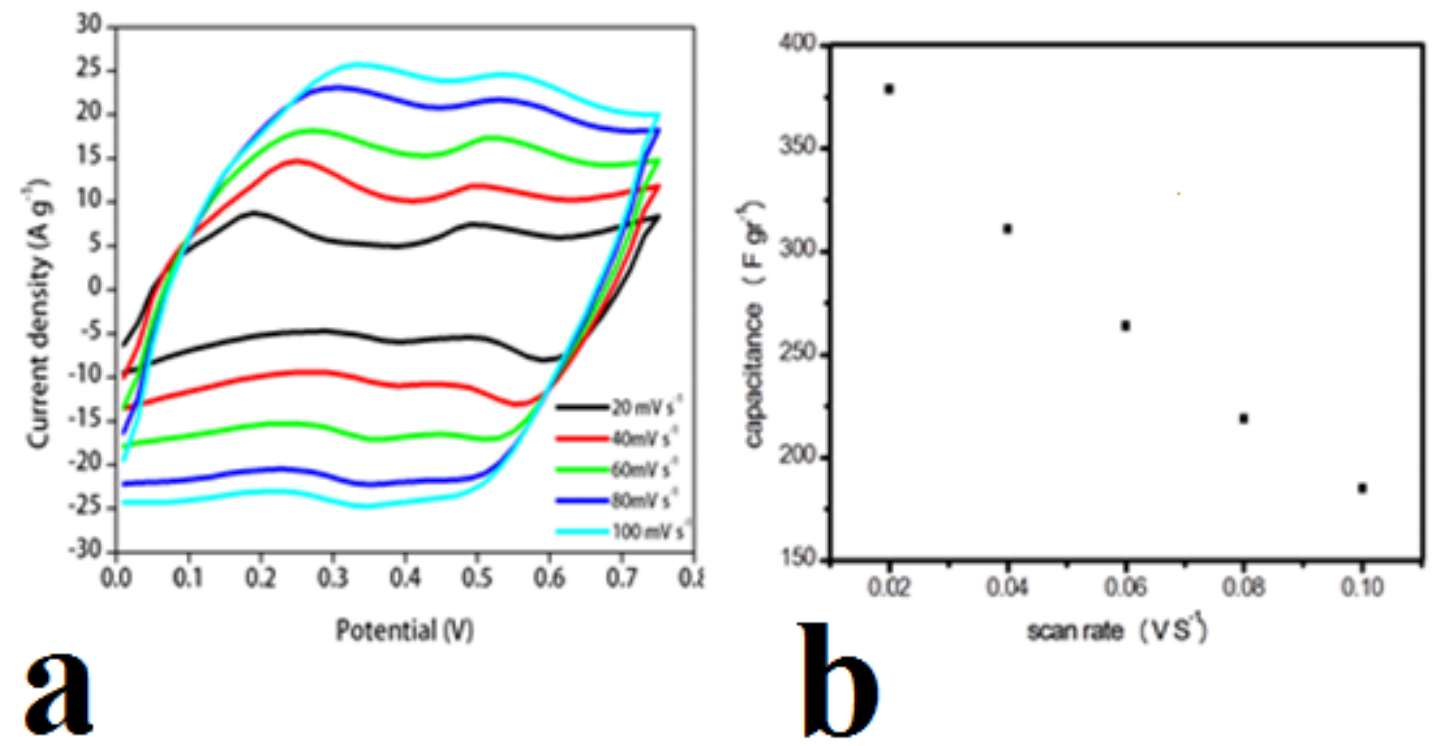

Fig. 3. (a) cyclic voltammograms of the MWCNT-PANI electrode measured in $0.5 \mathrm{M} \mathrm{H}_{2} \mathrm{SO}_{4}$ at different scan rates $20 \mathrm{mV} \mathrm{s}^{-1}$ to $100 \mathrm{mV} \mathrm{s}^{-1}$, and (b) the dependence of the capacitance of the electrode on the applied scan rate.

Fig. 4 shows the EIS of the MWCNT/PANI composite films containing $0.8 \mathrm{wt} \%$ of MWCNT in $0.5 \mathrm{M} \mathrm{H}_{2} \mathrm{SO}_{4}$ solution measured at $0.4 \mathrm{~V}$. The semicircle portion, observed at higher frequencies, corresponds to the electron transfer limited process, whereas the linear part is characteristic of the lower frequencies range and represents the diffusion al limited electron transfer process. It shows that the charge-transfer complex resistance of the MWCNT/PANI composite film is smaller than that of the pure PANI which is caused by formation of the charge-transfer complex between the uniform dispersion MWCNT and the PANI matrix of the composite, lowers defect density and lowers the resistance.[2] 


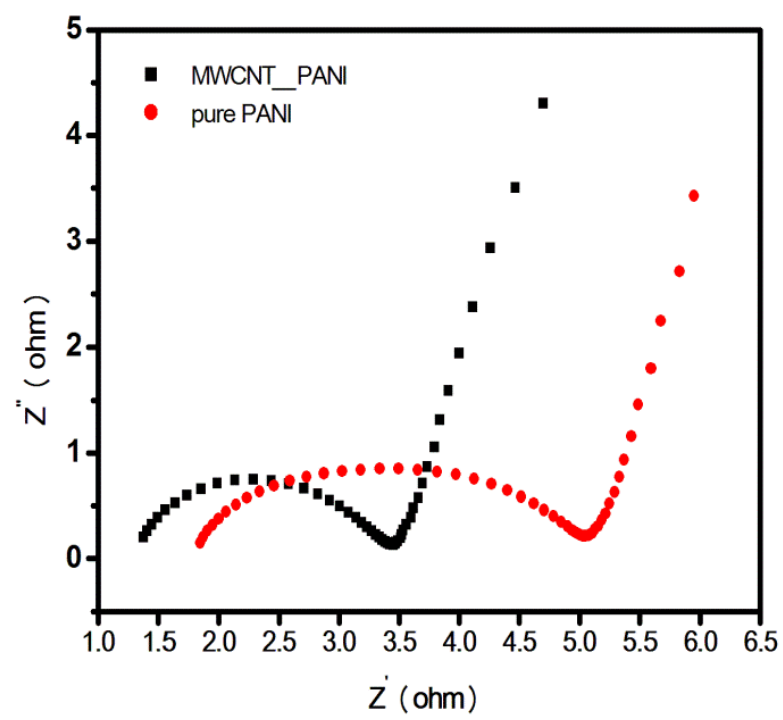

Fig.4. Nyquist plots of the pure PANI film and the MWCNT/PANI composite film.

\section{Conclusions}

The MWCNT/PANI composite films were synthesized successfully by in-situ electrochemical polymerization of aniline containing well-dissolved MWCNT. The MWCNT/PANI composite electrodes showed better capacitive characteristics than the pure PANI electrode. Compared with the pure PANI film, the MWCNT/PANI composite film was a highly porous film. MWCNT has an obvious improvement effect which makes the composite films more active sites for faradic reaction, larger SC, lower resistance than pure PANI and facilitates the chargetransfer of the composite films.

\section{References}

[1] C.Peng, Sh.Zhang, D.Jewell, George Z. Che "Carbon nanotube and conducting polymer composites for supercapacitors" Progress in Natural Science 18,777-788, 2008.

[2]J.Zhang, L.Kong, B.Wang, Yong-Chun Luo, " In-situ electrochemical polymerization of multi-walled carbon nanotube/polyaniline composite films for electrochemical supercapacitors" Synthetic Metals 159, 260-266, 2009.

[3] X.Wang, J.Deng, X. Duan, Dong Liu, J. Guo and Peng Liu " Crosslinked Polyaniline Nano rods with Improved Electrochemical Performance as Electrode Material for Supercapacitor " Mater. Chem A, 12, 2-22, 2014.

[4] W. Xing, Sh. Zhuo, H.Cui “ Enhanced electrochemical properties of polyaniline-coated multiwall carbon nanotubes" J Porous Mater ,15, 647-651, 2008. 
[5] B. Dong, Ben-Lin He, Cai-Ling Xu, Hu-Lin Li "Preparation and electrochemical characterization of polyaniline/multi-walled carbon nanotubes composites for supercapacitor" Materials Science and Engineering B, 143,7-13, 2007. 